\title{
Adaptive Evolution of Leptin in Heterothermic Bats
}

\author{
Lihong Yuan ${ }^{1,2}$, Xudong Zhao ${ }^{3}$, Benfu Lin ${ }^{1}$, Stephen J. Rossiter ${ }^{4}$, Lingjiang He ${ }^{5}$, Xueguo Zuo ${ }^{5}$, Guimei \\ $\mathrm{He}^{5}$, Gareth Jones ${ }^{6}$, Fritz Geiser ${ }^{7}$, Shuyi Zhang ${ }^{5 *}$
}

1 South China Institute of Endangered Animals, Guangzhou, China, 2 Key Laboratory of Marine Bio-resources Sustainable Utilization, Key Laboratory of Applied Marine Biology of Guangdong Province, South China Sea Institute of Oceanology, Chinese Academy of Sciences, Guangzhou, China, 3 Institute of Zoology, Chinese Academy of Sciences, Beijing, China, $\mathbf{4}$ School of Biological and Chemical Sciences, Queen Mary University of London, London, United Kingdom, $\mathbf{5}$ Institute of Molecular Ecology and Evolution, Institutes for Advanced Interdisciplinary Research, East China Normal University, Shanghai, China, 6 School of Biological Sciences, University of Bristol, Bristol, United Kingdom, $\mathbf{7}$ Center for Behavioural and Physiological Ecology, Zoology, University of New England, Armidale, Australia

\begin{abstract}
Heterothermy (hibernation and daily torpor) is a key strategy that animals use to survive in harsh conditions and is widely employed by bats, which are found in diverse habitats and climates. Bats comprise more than $20 \%$ of all mammals and although heterothermy occurs in divergent lineages of bats, suggesting it might be an ancestral condition, its evolutionary history is complicated by complex phylogeographic patterns. Here, we use Leptin, which regulates lipid metabolism and is crucial for thermogenesis of hibernators, as molecular marker and combine physiological, molecular and biochemical analyses to explore the possible evolutionary history of heterothermy in bat. The two tropical fruit bats examined here were homeothermic; in contrast, the two tropical insectivorous bats were clearly heterothermic. Molecular evolutionary analyses of the Leptin gene revealed positive selection in the ancestors of all bats, which was maintained or further enhanced the lineages comprising mostly heterothermic species. In contrast, we found evidence of relaxed selection in homeothermic species. Biochemical assays of bat Leptin on the activity on adipocyte degradation revealed that Leptin in heterothermic bats was more lipolytic than in homeothermic bats. This shows that evolutionary sequence changes in this protein are indeed functional and support the interpretation of our physiological results and the molecular evolutionary analyses. Our combined data strongly support the hypothesis that heterothermy is the ancestral state of bats and that this involved adaptive changes in Leptin. Subsequent loss of heterothermy in some tropical lineages of bats likely was associated with range and dietary shifts.
\end{abstract}

Citation: Yuan L, Zhao X, Lin B, Rossiter SJ, He L, et al. (2011) Adaptive Evolution of Leptin in Heterothermic Bats. PLoS ONE 6(11): e27189. doi:10.1371/ journal.pone.0027189

Editor: Brock Fenton, University of Western Ontario, Canada

Received July 8, 2011; Accepted October 11, 2011; Published November 16, 2011

Copyright: (c) 2011 Yuan et al. This is an open-access article distributed under the terms of the Creative Commons Attribution License, which permits unrestricted use, distribution, and reproduction in any medium, provided the original author and source are credited.

Funding: This work was funded by a grant awarded to SZ under the Key Construction Program of the National "985" Project and " 211 " Project. The funders had no role in study design, design, data collection and analysis, decision to publish, or preparation of the manuscript.

Competing Interests: The authors have declared that no competing interests exist.

* E-mail: syzhang@bio.ecnu.edu.cn

\section{Introduction}

The ability of heterothermic mammals to regulate or vary their body temperature $\left(\mathrm{T}_{\mathrm{b}}\right)$ in response to changes in ambient temperature $\left(\mathrm{T}_{\mathrm{a}}\right)$ and energy requirements is considered key to their evolutionary success, allowing them to survive in adverse climates and during periods when food is scarce [1,2]. Heterothermy contrasts with homeothermy, characterized by a more or less constant and high $\mathrm{T}_{\mathrm{b}}$ largely irrespective of $\mathrm{T}_{\mathrm{a}}$ [3]. Heterothermy in mammals has been categorized as either daily torpor or prolonged multi-day torpor (hibernation), which the duration of torpor bouts last for less than $24 \mathrm{~h}$ and $\mathrm{T}_{\mathrm{b}}$ maintained around $15^{\circ} \mathrm{C}-25^{\circ} \mathrm{C}$ in the former whereas between $100 \mathrm{~h}$ to $1000 \mathrm{~h}$ and $\mathrm{T}_{\mathrm{b}}$ of $0-10^{\circ} \mathrm{C}$ in the latter [4]. Comparative data show that heterothermy is employed for energy conservation by members of at least ten orders of mammals, including monotremes, four marsupial orders, carnivores, rodents, eulipotyphlans (insectivores), bats and primates [5,6,7,8,9].

Bats (order Chiroptera) number over 1100 species and are among the most geographically widespread of mammals [10]. Most bat families contain species that can enter daily torpor, whereas hibernation is confirmed in at least seven families
$[1,11,12]$. Hibernating species of bats (some members of the superfamilies Emballonuroidea, Vespertilionoidea and Rhinolophoidea) are distributed in cool temperate or subtropical regions, whereas homeothermic bats are mainly tropical or subtropical [13]. Despite these differences in distribution and thermoregulatory patterns, little is known about the evolutionary history of heterothermy in bats. This is complicated further because phylogenetic reconstructions indicate that hibernation occurs in several branches across the mammalian tree [14]. However, currently there is no obvious molecular marker that can be reliably associated with hibernation.

All heterothermic mammals, including hibernating bats require efficient use of adipose tissue because they rely on fat as the primary energy source during periods of torpor, which in turn permits survival of exceedingly low $\mathrm{T}_{\mathrm{a}}$ and scarce food [7]. Hibernators must have an accurate and efficient way to control lipolysis, not only to control the time point of metabolizing fat for heat production during periodic rewarming, but also to extend the energy supply throughout long winters. The hormone Leptin has been shown to be involved in lipid metabolism and the provision of energy via lipolysis [15]. Metabolism and food intake in hibernating species appears to be regulated by periodic changes in 
the level of serum Leptin and other related molecules $[16,17,18,19,20]$. It was recently suggested that exposure to cold environments has driven the evolution of Leptin by selected site substitution in one group of lagomorphs [21]. Such studies implicate Leptin in the control of thermogenesis, crucial for successful hibernation, and therefore the evolution of Leptin in hibernators is also likely to be driven by natural selection.

To tease apart the molecular evolution of bat Leptin, and explore the possible evolutionary history of heterothermy (hibernation and torpor) in bats, we employed three approaches: (i) we compared thermoregulation in four tropical/subtropical species, two Old World fruit bats and two insectivorous bats, thought to differ in their capacity for expression of heterothermy; (ii) we reconstructed the ancestral states and undertook evolutionary and phylogenetic analyses of the Leptin gene in a range of heterothermic and homeothermic bat lineages (see Table S1); and (iii) we expressed two recombinant proteins of bat Leptin with glutathione-Stransferase (GST) tags in E. coli cells and used them for bioactivity assays in vitro to compare the effect of Leptin on adipocyte lipolysis between heterothermic and homeothermic bats. As lipolysis is a process that provides chemical energy by adipocyte degradation, the lipolytic activity of Leptin is reflected by adipocyte viability, which can be assessed by the reduction of the number of adipocytes, or via quantifying release of Lactase dehydrogenase $(\mathrm{LDH})$ into the media.

\section{Materials and Methods}

\section{Ethics statement}

The physiological experiment and sampling were approved by the Administrative Panel on Laboratory Animal Care of Institute of Zoology, Chinese Academy of Sciences, and accord with the guidelines for the National Care and Use of Animals approved by the National Animal Research Authority (approval ID 20080209).

\section{Study of thermoregulatory ability}

Four similar-sized species of bats (2 pteropodids: Cynopterus sphinx and Eonycteris spelaea, 1 hipposiderid: Hipposideros armiger and 1 vespertilionid: Scotophilus heathii) from subtropical and tropical zones were chosen for the thermoregulatory study. All bats were collected in the southeast of China, and were housed individually in separate cages and supplied with food/water and a 12:12 light: dark cycle (lights on from 06:00 to 18:00 h). A telemetry $\mathrm{T}_{\mathrm{b}}$ transmitter (DSI, USA) was surgically implanted and used to record the $\mathrm{T}_{\mathrm{b}}$ responsing to $\mathrm{T}_{\mathrm{a}}$ which change from $25^{\circ} \mathrm{C}$ to $5^{\circ} \mathrm{C}$. One-way analysis of variance (ANOVA) was used for comparison of mean $\mathrm{T}_{\mathrm{b}}$ of each individual of the four species at the three $\mathrm{T}_{\mathrm{a}} \mathrm{s}$ measured (see Text S1).

\section{Molecular phylogenetic analysis}

To assess the evolutionary history of Leptin in heterothermic bats, we carried out analyses of molecular evolution in a range of heterothermic and homeothermic bat lineages.

Reconstruction of ancestral characters. Based on the composite species tree [22,23], we reconstructed the ancestral states with binary data (heterothermy vs. homeothermy) and multistate data (hibernation, torpor, homeothermy), using the parsimony and likelihood methods in MESQUITE2.1 [24], to infer the lineages which are potentially related with the evolution of heterothermy.

Sequencing and database searches. The Leptin complete CDS or exon 3 were cloned and sequenced from 19 bat species, including six species of the family Pteropodidae: Eidolon helvum, Eonycteris spelaea, Rousettus leschenaultii, Dobsonia viridis, Cynopterus sphinx,
Pteropus giganteus; and 13 species from other families: Taphozous melanopogon (Emballonuridae), Miniopterus fuliginosus (Miniopteridae), Scotophilus heathii, Myotis ricketti (Vespertilionidae), Chaerephon plicatus, Tadarida teniotis (Molossidae), Pteronotus parnellii (Mormoopidae), Artibeus gnomus, Anoura geoffroyi, Carollia brevicauda (Phyllostomidae), Hipposideros armiger (Hipposideridae), Rhinolophus ferrumequinum (Rhinolophidae), Rhinopoma microphyllum (Rhinopomatidae) (see Text S1 and Table S2). Moreover, the complete Leptin sequences of a further 27 mammal species were obtained from the NCBI Nucleotide collection (nr) and Whole-genome shotgun (wgs) databases [25], including six primates: Homo sapiens, Pan troglodytes, Pongo abelii, Macaca mulatta, Otolemur garnettii and Microcebus murinus; two rodents: Rattus norvegicus and Mus musculus; eight lagomorphs: Lepus oiostolus, Oryctolagus cuniculus, Ochotona dauurica bedfordi, Ochotona annectens, Ochtona nubrica, Ochotona curzoniae, Ochotona cansus cansus and Ochotona princeps; three artiodactyls: Sus scrofa, Bos taurus and Capra hircus; one perissodactyl: Equus caballus; three carnivores: Felis catus, Ursus thibetanus japonicus and, Canis lupus familiaris; one bat: Myotis lucifugus; one proboscidean: Loxodonta africana; one marsupial: Monodelphis domestica, and one monotreme: Ormithorhynchus anatinus.

Evolutionary analyses. Nucleotide sequences were aligned by using ClustalX1.81 [26], and translated in MEGA3.1 [27]. The best-fit model of molecular evolution were determined by Modeltest 3.7 [28] and the phylogenetic trees of Leptin were constructed by MrBayes 3.1.1 [29].

To identify the variable selective pressures in the Leptin, both across the tree and along specific lineages, we carried out the CODEML program analyses in the PAML package: a free-ratio model, site-specific models, a branch-specific model (two-ratio), and a branch-site model (Model A) [30] (Text S1). We also included published sequence data from the lagomorph group of pikas, in which molecular evolution of Leptin has previously been shown to be linked to cold environmental stresses [21].

To resolve further the distribution in selective pressures on specific functional domains of Leptin among different clades, we presented Nei and Gojobori [31] substitution rates $\left(d_{\mathrm{N}}\right.$ and $d_{\mathrm{S}}$ per site and the $d_{\mathrm{N}} / d_{\mathrm{S}}$ ratio) as sliding windows (window $=45$ codons, step $=3$ codons) for exon 3 using the software SWAAP 1.0.2 [32]. This was repeated for three groups of taxa: 21 placental mammals excluding $U$. thibetanus japonicusand, M. murinus and M. musculus, 14 heterothermic bats, 6 homeothermic bats.

To test whether the amino acid replacements in the Leptin protein of heterothermic bats are likely to have an impact on protein function, we undertook a multivariate based analysis of protein physicochemical properties using the program MAPP [33]. For this analysis, amino acid sequences of Leptin exon 3 orthologues of 27 homeothermic placental mammals and 14 heterothermic bats were aligned, respectively, and weighted by known phylogenetic relationships. From the variation in the dataset, the physicochemical constraint of each site was estimated based on hydropathy, polarity, charge, volume and free energy, and to predict the impact of all possible replacements seen in hibernating bats (MAPP score).

Bio-activity assay of Leptin on thermoregulation. Leptin can directly act on adipocyte lipolysis in vitro and in vivo [34,35]. To validate differences in activity of Leptin lipolysis between heterothermic and homeothermic bats, the recombinant Leptin proteins of a hibernating species (M. fuliginosus) and a homeothermic species $(R$. leschenaultii) were fused with glutathione-S-transferase (GST) tags and expressed in E. coli cells. Then, the lipolytic activity of GST-Leptin was measured by the inhibitory effect on cell viability and cytotoxicity on adipocytes.

Leptin coding sequences of the two bats, excluding the first 21 amino acids signature domain were amplified by PCR (see Text 
S1). 3T3-L1 mouse embryo fibroblasts (adipose-like cells, ATCG) were cultured according to the methods described by Hemati et al [36], and used for Leptin activity assay. The effect of Leptin on adipoctytes is reflected by cell viability. One assessment of cell viability is to assess the number of viable cells based on the mitochondrial-dependent reduction of 3-(4,5-dimethylthiazol-2-yl) -2,5-diphenyl tetrazolium bromide (MTT) method. Another is to measure the Leptin cytotoxicity by Lactate dehydrogenase (LDH) assay using the CytoTox-ONETM assay kit (Promega), as the presence of $\mathrm{LDH}$ in media relative to its activity in cells is used as criterion of direct cytotoxicity. In our study, 3T3-L1 cells were incubated with bat GST-Leptin $\left(10^{-6} \mathrm{M}\right)$ at $24 \mathrm{~h}$ or $48 \mathrm{~h}$. Experiments were repeated three times with four to six replicates for each treatment. Details of procedures followed are described by Ambati et al. [34], and a $t$-test was used to compare the means of the two bat species.

\section{Results}

\section{Thermoregulatory study}

Cynopterus sphinx and Eonycteris spelaea (Pteropodidae) did not enter torpor or hibernation at all $\mathrm{T}_{\mathrm{a}} \mathrm{s}$ measured (Figure 1). The $\mathrm{T}_{\mathrm{b}}$ of individuals was little affected by $\mathrm{T}_{\mathrm{a}}$ and mean $\mathrm{T}_{\mathrm{b}}$ was $36.4 \pm 0.2^{\circ} \mathrm{C}$ for C. sphinx $(\mathrm{N}=6, \mathrm{n}=36)$ and $36.8 \pm 0.5^{\circ} \mathrm{C}$ for E. spelaea $(\mathrm{N}=6$, $\mathrm{n}=36)$. In contrast, Scotophilus heathii and Hipposideros armiger entered torpor, at $\mathrm{T}_{\mathrm{a}} 15^{\circ} \mathrm{C}$ and $5^{\circ} \mathrm{C}$. At $\mathrm{T}_{\mathrm{a}} 15^{\circ} \mathrm{C}$ and $5^{\circ} \mathrm{C}$, the average $\mathrm{T}_{\mathrm{b}}$ were $26.29 \pm 1.58^{\circ} \mathrm{C}$ and $8.34 \pm 1.16^{\circ} \mathrm{C}$ for $S$. heathii, respectively $\left(\mathrm{F}_{(2,15)}=133.58 ; \mathrm{P}<0.0001\right.$; One Way ANOVA); at the same $\mathrm{Ta}$ condition, $\mathrm{Tb}$ of $H$. armiger were $21.78 \pm 0.46^{\circ} \mathrm{C}$ and $12.81 \pm 0.77^{\circ} \mathrm{C}\left(\mathrm{F}_{(2,15)}=261.6 ; \mathrm{P}<0.0001\right.$; One Way ANOVA $)$, respectively. When the $\mathrm{T}_{\mathrm{a}}$ decreased, C. sphinxand E. spelaea remained active and ate more food than at $\mathrm{T}_{\mathrm{a}} 25^{\circ} \mathrm{C}$; no sign of discomfort was observed. In contrast, torpor in $S$. heathii and $H$. armiger at $\mathrm{T}_{\mathrm{a}} 15^{\circ} \mathrm{C}$ was observed on both measurement days, but bats showed full or partial rewarming daily; at $\mathrm{T}_{\mathrm{a}} 5^{\circ} \mathrm{C}$ both species hibernated without rewarming over the two days.

\section{Evolutionary and selective pressure analysis}

Tracing character history of ancestors indicated that bat heterothermy mainly evolved in three lineages (Figure 2 and
Figure S1). We hypothesized that, if Leptin is the key of bat heterothermic evolution, natural selected pressure on Leptin may be detected in these lineages. To test this hypothesis, we cloned the complete Leptin CDS from five bat species and Leptin exon 3 from 14 bat species (accession numbers GU230829- GU230833 and GU230835-GU230848) are listed in Table S3.

The Leptin gene tree based on the complete CDS, constructed by MrBayes 3.1.1 [29] according to the best-fit model (K80+G) determined by Modeltest 3.7 [28] is shown in Figure S2. The tree topology was consistent with that of previous multigene phylogenies of mammals [23], and this topology was then constrained in subsequent estimates of substitution rates. The free-ratio model showed that the $\omega$ ratio was considerably higher in the ancestor of the strepsirrhines (non-tarsier prosimians) $\left(\omega=999.000, \mathrm{~N}^{*} \mathrm{dN}=4.3\right.$, $\left.\mathrm{S}^{*} \mathrm{dS}=0\right)$, the hominid lineage $\left(\omega=2.048, \mathrm{~N}^{*} \mathrm{dN}=6.1, \mathrm{~S}^{*} \mathrm{dS}=0\right)$, the chiropteran ancestor $(\omega=616.224, \mathrm{~N} * \mathrm{dN}=5, \mathrm{~S} * \mathrm{dS}=0)$, the yangochiropteran lineage $\left(\omega=1.225, \mathrm{~N}^{*} \mathrm{dN}=13.1, \mathrm{~S} * \mathrm{dS}=3.5\right)$ and the rhinolophoid lineage $\left(\omega=1.364, \mathrm{~N}^{*} \mathrm{dN}=70.4, \mathrm{~S} * \mathrm{dS}=17.0\right)$ than in other lineages, and the $\omega$ ratio in the pika lineage was 0.544 (Figure S2). The LRT statistic of the free-ratio model vs. M0 was much greater than critical values from a $\chi^{2}$ distribution with $2 \Delta \ell=129.86$ $(\mathrm{P}<0.001$ and d.f. $=60)$ (Table S4). Branch-specific analysis confirmed the selective pressure on these specific lineages with $\mathrm{P}<0.001$ (Table S4). Site-specific analyses indicated that the selective pressure was highly variable among sites, as $\mathrm{M} 0$ was rejected by a big margin compared with $\mathrm{M} 3$ with $2 \Delta \ell=180.28(\mathrm{P}<0.001$ and d.f. $=4)$, and 15 natural selected sites were identified (Table S4). In addition, model M2a and M8 suggested that $0.78 \%$ and $1.23 \%$ of sites were under positive selection with $\omega>1$, although the LRT of M8/M8a and $\mathrm{M} 2 \mathrm{a} / \mathrm{M} 1 \mathrm{a}$ did not achieve significance (Table S4).

To improve our chances of detecting adaptive changes and identifying informative sites within the Chiroptera, we cloned and sequenced exon 3 in an additional 14 bat species. The phylogenetic tree of Leptin exon 3 (TrNef+G) was again consistent with multigene phylogenies of mammals and bats [22,23]. From the exon 3 data, the free-ratio model showed that $\omega$ was higher in the ancestral branch to all bats and in the Primate lineage (no synonymous changes) than in other lineages, with $\omega=0.581$ in the pika lineage. Within the primate lineage, $\omega$ ratios of two lineages, the Strepsirrhini $\left(\omega=999.8, \mathrm{~N}^{*} \mathrm{dN}=3.2, \mathrm{~S} * \mathrm{dS}=0\right)$ and Homini-

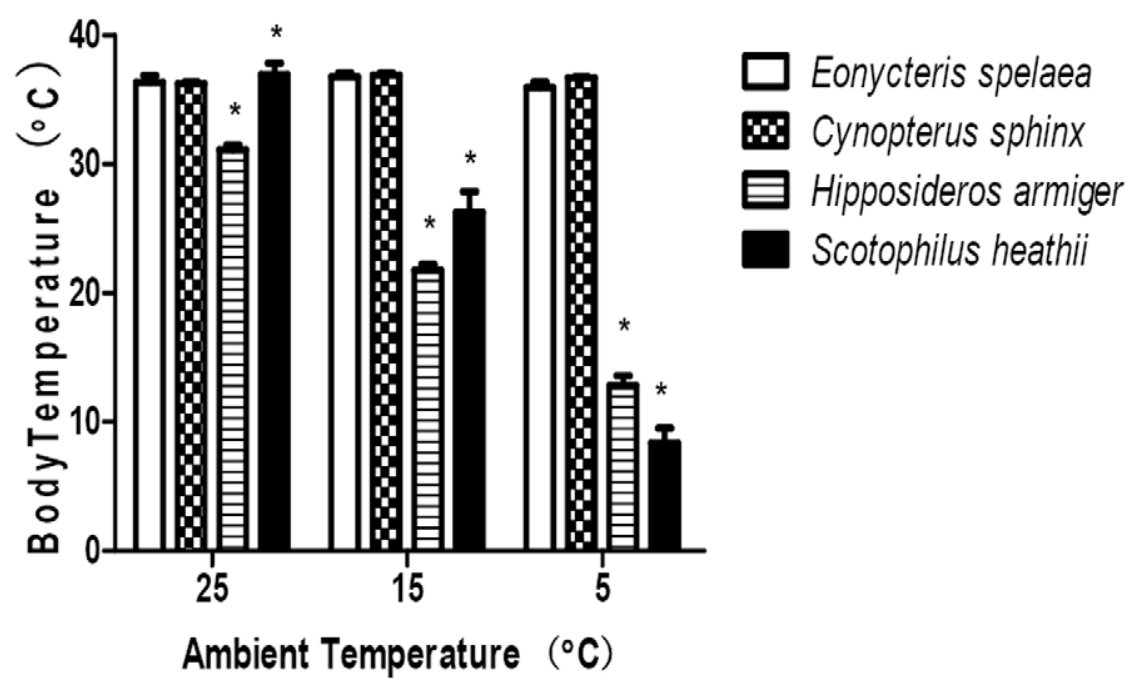

Figure 1. Comparison of body temperature of four bats as a function of ambient temperature. Eonycteris spelaea ( $\mathrm{N}=6), \mathrm{Cynopterus}$ sphinx $(\mathrm{N}=6)$, Hipposideros armiger $(\mathrm{N}=6)$ and Scotophilus heathii $(\mathrm{N}=6)$. The $\mathrm{SE}$ is indicated by the error bars. ${ }^{*} \mathrm{P}<0.0001$. doi:10.1371/journal.pone.0027189.g001 


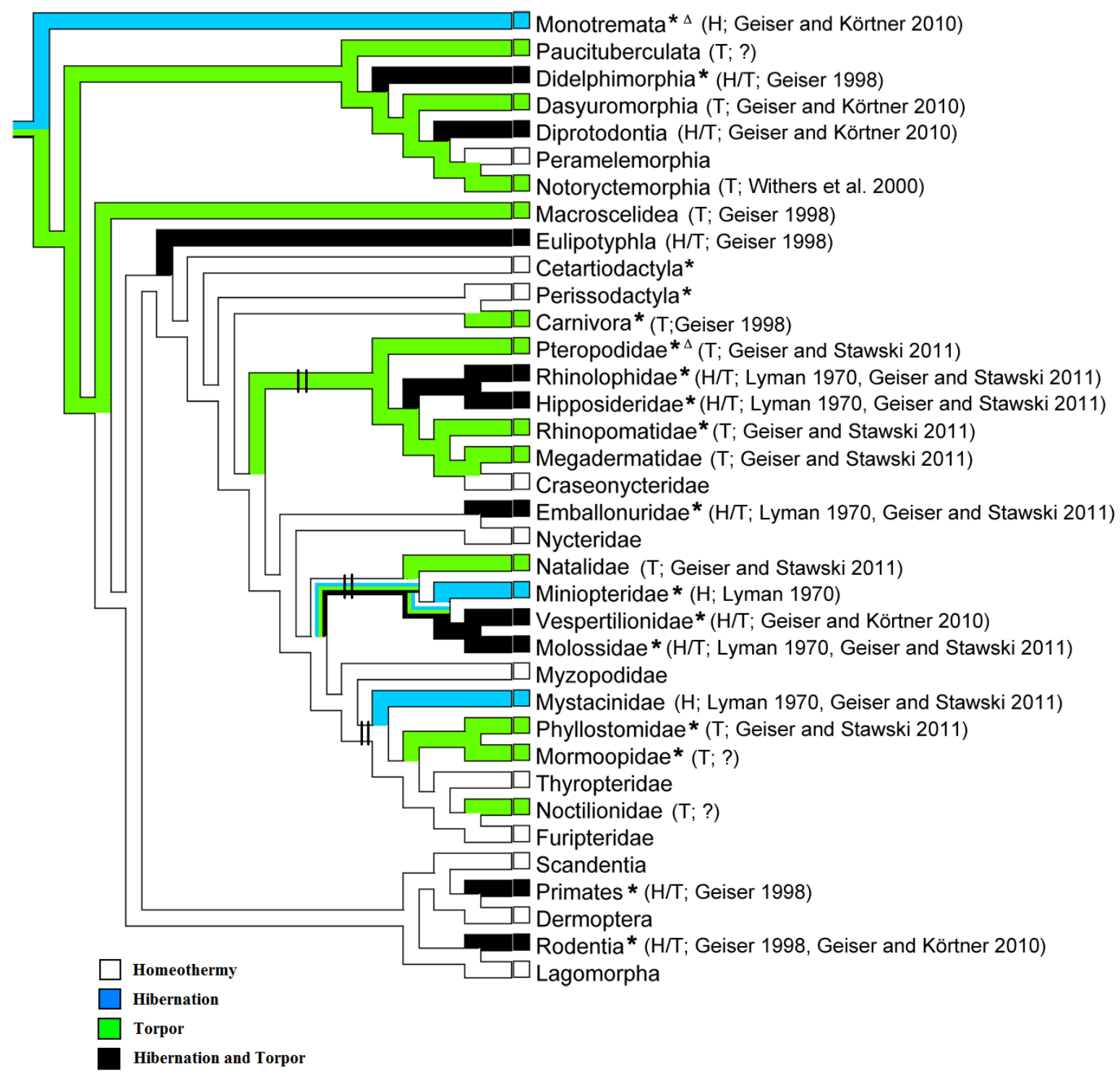

Figure 2. Reconstruction of ancestral states. Multistate data of homeothermic and heterothermic (hibernation and/or torpor) states are mapped onto the composite species tree (Teeling et al. 2005 and Nishihara, Hasegawa, and Okada 2006). Branches show the species of family are homeothermic (white), hibernators (blue), torpor (green), or hibernators and torpor (black). *: some species of this family were test in this study; $\Delta$ : species studied here are homeothermic. ?: some species of this family are likely heterothermic. Vertical bars on branches represent the lineages which are potentially related with the evolution of bat heterothermy. doi:10.1371/journal.pone.0027189.g002

$\operatorname{dea}\left(\omega=999.000, \mathrm{~N}^{*} \mathrm{dN}=6.3, \mathrm{~S} * \mathrm{~d} \mathrm{~S}=0\right)$, are $>1$. Moreover, the $\omega$ ratio of the Yangochiroptera $(\omega=974.2, \mathrm{~N} * \mathrm{dN}=4.6, \mathrm{~S} * \mathrm{dS}=0)$ and Rhinolophoidea $(\omega=999.0, \mathrm{~N} * \mathrm{dN}=14.3, \mathrm{~S} * \mathrm{dS}=0)$ were greater than those of the Yinpterochiroptea $(\omega=0.151)$ and Pteropodidae $(\omega=0.760)$, respectively (Figure 3$)$. The LRT statistics of the free-ratio model vs. M0 and M3 vs. M0 achieved significance (Table S5). This result is consistent with the analysis based on Leptin complete CDS and indicates natural selection drove the evolution of Leptin in heterothemic placental mammals, especially in bats (Table S5). Then, the selected pressure on the rhinolophoid lineage was verified by the branch-specific analysis (Table S5). Moreover, data of the free-ratio analysis also indicated the potential of selected pressure $(\omega>1)$ on the sub-branchs within pteropodidae, which are homeothermy (Figure 3). Thus, branchsite analysis (Model A) was performed and data showed that the
LRT statistic of homeothermic bat lineage (the pteropodid) passed test I $(\mathrm{LRT}=10.57$, d.f. $=3, \mathrm{P}<0.05)$, but did not pass test II $(\mathrm{LRT}=1.40$, d.f. $=1, \quad \mathrm{P}>0.05)$, suggesting potential relaxed selection on homeothermic bats (Table S5).

After removing all gaps (12 bp), the alignment of Leptin exon 3 consisted of $330 \mathrm{bp}$ encoding 110 amino acids. A sliding window revealed differences in substitution rates among specific groups. The $\omega$ values $\left(d_{\mathrm{N}} / d_{\mathrm{S}}\right)$ of two groups, heterothermic bats and homeothermic bats, were clearly higher than those of 21 other homeothermic placental mammals (Figure 4). For heterothermic bats, most parts of the Leptin exon 3 showed elevated $\omega$ ratios, especially in the $\mathrm{AB}$ loop and helix $\mathrm{D}$ regions, supporting maximum-likelihood based analyses. Moreover, $\omega$ values of the $5^{\prime}$ terminal of $\mathrm{AB}$ loop and the $5^{\prime}$ terminal helix $\mathrm{E}$ in homeothermic bats were higher than in heterothermic bats. 


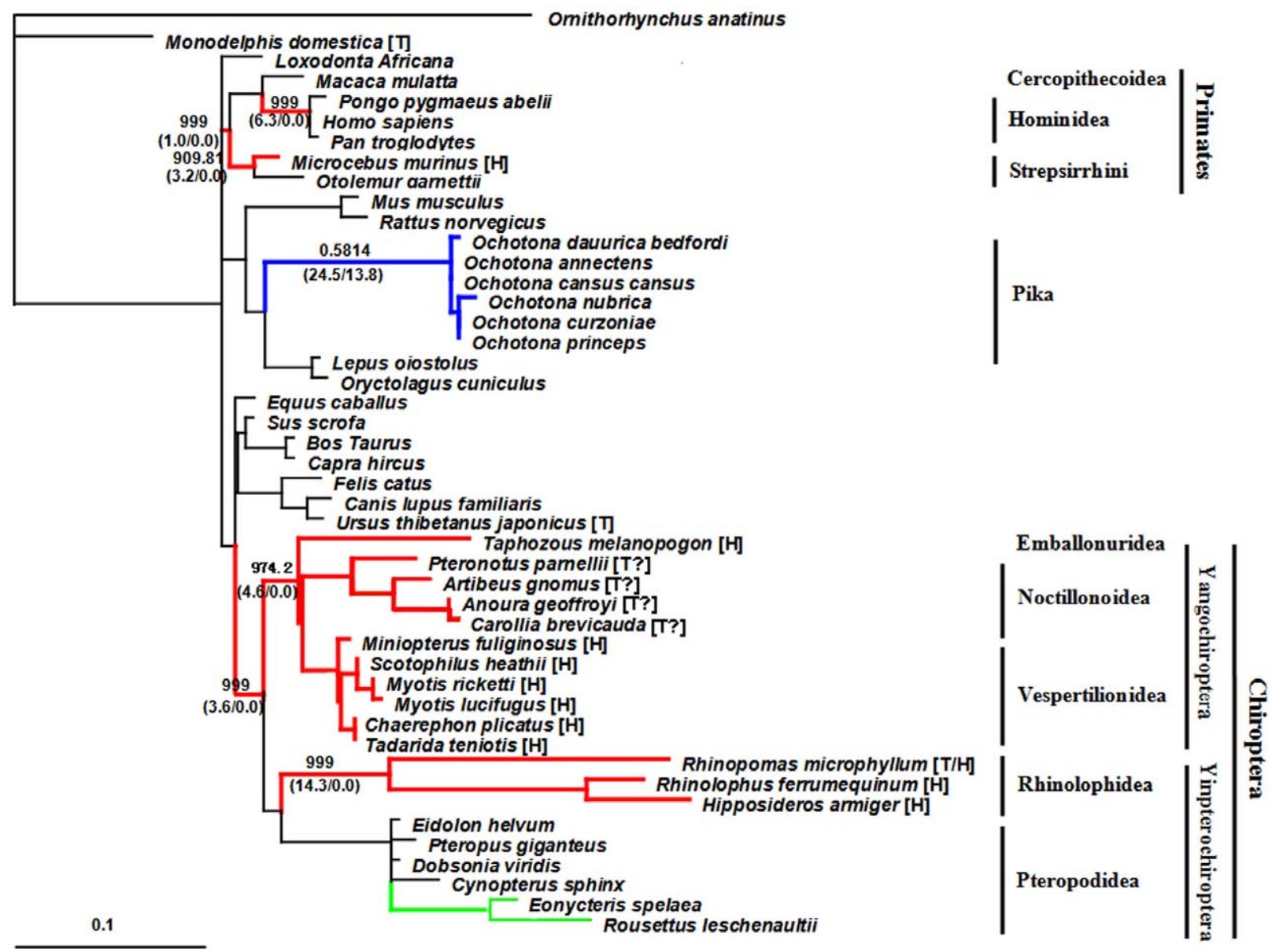

Figure 3. The phylogenetic gene tree of Leptin. The gene tree was based on nucleotide sequences of Leptin exon 3 and used for the codon maximum likelihood analysis. The $\omega$ ratios and numbers of non-synonymous substitution $\left(N^{*} d N\right)$ and synonymous substitution ( $\left.S^{*} d S\right)$ of some specific lineages were shown in bold and in bracket. The scale bar of " 0.1 " means 0.1 nucleotide substitution per site. [T], torpor; [H], hibernator. Lineages with $\omega>1$ were shown in red (heterothermy) and green (homeothermy). The Pika lineage as control was shown in blue. ?: it is likely heterothermic.

doi:10.1371/journal.pone.0027189.g003

Twenty-nine amino acid variants that were inferred to have large functional effects by multivariate analyses of physicochemical properties, were found in heterothermic bats (mean MAPP score $=21.9 \pm 0.23(\mathrm{SE})$; range 7.58-36.58) (Table S6). Of these, seven sites were located in the $\mathrm{AB}$ loop, five in the helix $\mathrm{B}$, eight in the helix $\mathrm{C}$, two in the $\mathrm{CD}$ loop, one in the helix D, and six in the helix E.

\section{Lipolysis activity assay of bat Leptin}

The substitutions of nucleotides or amino acids may change the spatial structure and affect the function of proteins. Therefore, recombinant GST-Leptin $(\sim 42 \mathrm{kDa})$ of two bat species, Miniopterus fuliginosus (hibernating) and Rousettus leschenaultii (homeothermic), was obtained and identified by SDS-PAGE and Western-blot analyses (Figure S3) to compare the functional difference between heterothermic and homeothermic Leptin.

Leptin MTT activity assays, carried out on 3T3-L1 preadipocytes, showed that the number of viable cells treated with heterothermic bat GST-Leptin was less than that of cells incubated with homeothermic bat GST-Leptin (Figure 5A). The proportion of living cells treated with $M$. fuliginosus Leptin decreased by $16.65 \%$, and was significantly lower $(\mathrm{P}=0.014)$ than that for $R$. leschenaultii Leptin (8.85\%). An LDH assay (to determine whether the decrease of cell viability was caused by the cytotoxic effect of bat Leptin) showed that after $48 \mathrm{~h}$, 3T3-L1 preadipocytes incubated with $10^{-6} \mathrm{M}$ bat Leptin showed a significantly higher $\mathrm{LDH}$ release $(\mathrm{P}=0.04 ; 6.97 \%)$ after treatment with $M$. fuliginosus Leptin than those with $R$. leschenaultii Leptin (1.57\%; Figure 5B). Consequently, bat Leptin, especially $M$. fuliginosus Leptin, can reduce cell viability and increase $\mathrm{LDH}$ release significantly, showing that the Leptin of the heterothemic bat ( $M$. fuliginosus) is more active in adipocyte degradation than that of the homeothermic bat $(R$. leschenaultii).

\section{Discussion}

The evolutionary history of mammalian heterothermy remains a controversial subject although it is crucial to the success of a large number of species. In bats, which colonized and are occupying vastly diverse habitats, evolution of heterothermy is especially intriguing given their biogeography and their large range of thermoregulatory abilities. A number of scenarios regarding the evolution of heterothermy in bats have been proposed [1,14]. If bats evolved in a temperate climate, hibernation is likely to be an ancestral trait that allowed bats to 


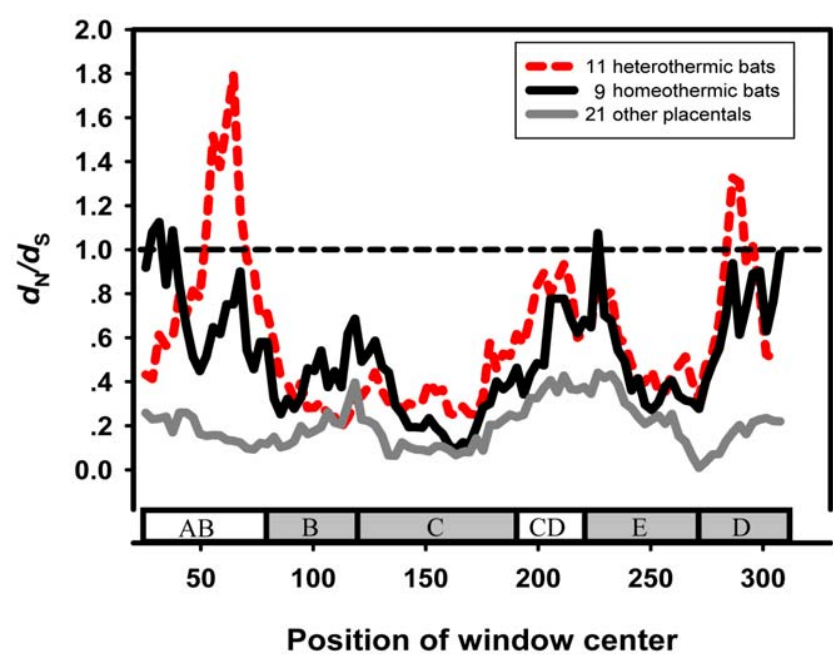

Figure 4. Sliding window analysis of Nei-Gojobori estimates. $d_{\mathrm{N}} / d_{\mathrm{S}}$ of 21 other placentals, 14 heterothermic bats and 6 hoemothermic bats (Figure 3, excluding Ursus thibetanus japonicus, Microcebus murinus, Mus musculus and the outgroups). In each case is compared to a gene schematic showing the loop (white) and helix (gray) domains, with the window size and step size were fixed at $45 \mathrm{bp}$ and $3 \mathrm{bp}$. The horizontal broken line identifies $\omega=1$.

doi:10.1371/journal.pone.0027189.g004

adapt to low environmental temperatures and limited food resources. As these lineages colonized tropical latitudes, the ability of hibernation was lost or was modified to become daily torpor [1]. Alternatively, if bats evolved in the tropics or subtropics and had the ability of daily heterothermy [37], hibernation in temperate species or homeothermy in tropical species are derived states [1]. A third possibility is that ancestral bats were homeothermic, although this seems unlikely given that homeothermy in extant bat taxa is relatively rare. Our data support the view of Lyman [1] that heterothermy in bats is an ancestral trait and was subsequently lost in now homeothermic bats. We provide physiological, molecular and biochemical evidence supporting this interpretation.

Our physiological data show clear differences in the thermoregulatory patterns between tropical/subtropical fruit bats and insectivorous bats when these were subjected to changes in $\mathrm{T}_{\mathrm{a}}$. Stable $\mathrm{T}_{\mathrm{b}}$ maintained at low $\mathrm{T}_{\mathrm{a}}$ in the former demonstrate that these fruit bats are homeothermic, whereas low $\mathrm{T}_{\mathrm{b}}$ and torpor bouts without arousals for at least 2 days at $\mathrm{T}_{\mathrm{a}} 5^{\circ} \mathrm{C}$ clearly show that these insectivorous bats have retained the ability to hibernate although they live in tropical/subtropical areas.

In the Chiroptera, most families include heterothermic species, including the New World frugivorous species and some Old World fruit bats $[1,38,39,40]$. According to the latest molecular phylogenetic data and fossil evidence, the common ancestor of bats originated during the Cenozoic Era around 64 million years ago (Mya) in Laurasia and diverged into two suborders (the Yangochiroptera and Yinpterochiroptera) [22]. Although some extant small species in the Pteropodidae (which originated 24 Mya) still keep the ability of daily torpor [40], most species are considered to be strictly homeothermic consistent with our data (Figure 1), likely because they are restricted to tropical/ subtropical climates and have specialized on a diet of fruit [13,22]. Together with the evolutionary history of the Chiroptera, the chronological distribution of heterothermy in bats, and their current geographic ranges, the most parsimonious assumption is that the ancestors of bats were heterothermic (Figure 2 and Figure S1). Subsequent changes in climate and associated shifts in

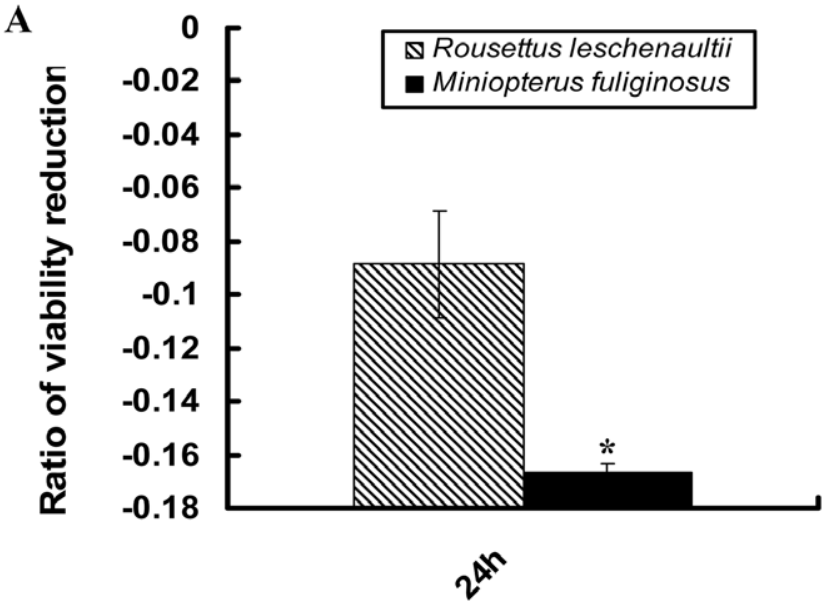

Incubation period

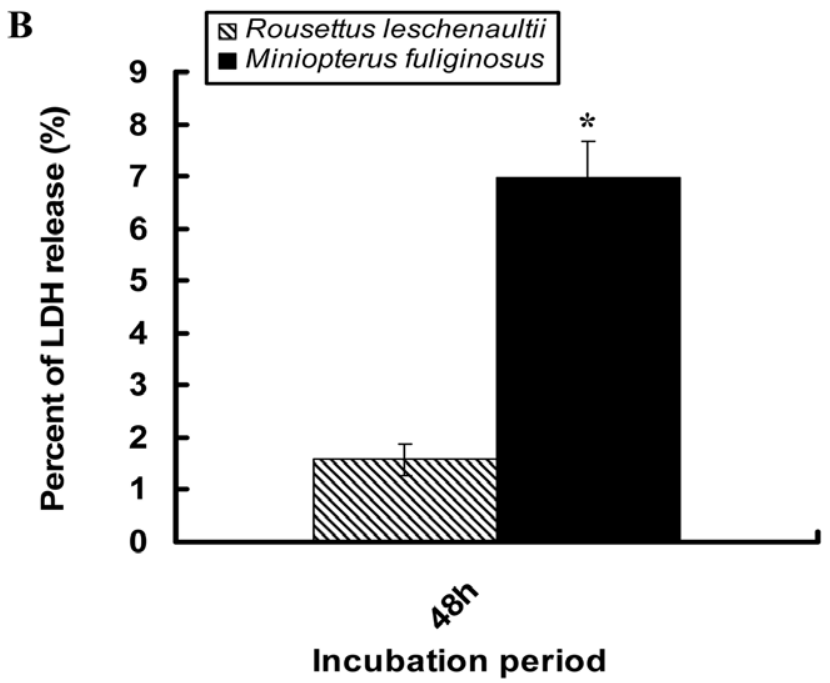

Figure 5. Activity assay of bat Leptin. Relative effect of bat Leptin on viability (MTT) $(A)$ and cytotoxicity of 3T3-L1 adipocytes (LDH) (B). 3T3-L1 cells were incubated with bat GST-Leptin $\left(10^{-6} \mathrm{M}\right)$ at $24 \mathrm{~h}$ or $48 \mathrm{~h}$. Cell viability and cytotoxicity were tested by the MTT and LDH assay with four to six replicates for each treatment and experiments were repeated three times. Data are means \pm SD. Asterisk indicates significant difference $(* \mathrm{P}<0.05 ; t$-test).

doi:10.1371/journal.pone.0027189.g005

distribution of species led to the secondary loss of heterothermy in most Old World fruit bats. By comparison, most temperate insectivorous bats and some tropical species have retained their ability to express torpor.

Our molecular phylogenetic analyses with Leptin suggest natural selection in heterothermic mammals (Primates and bats). This was achieved by testing the selection pressures upon Leptin within placental mammals only, and using the marsupials and monotremes as outgroups because the evolution of heterothermy appears to differ between placental mammals and other mammals [41]. The results of the codon maximum likelihood analysis based on Leptin complete CDS and Leptin exon 3 were identical and showed that the $\omega$ ratios of three lineages, Primates, Hominidea and Strepsirrhini, were $>1$, consistent with many previous studies $[42,43,44]$. Moreover, our results suggest that natural selection resulted in positive evolution of Leptin in the ancestors of bats and 
also in the heterothermic bats (Figure 3 and Figure S2). In contrast, Leptin in the homeothermic bats (pteropodid lineage) seems to be under relaxed selection, according to the LRT statistic of Model A (Table S5). These observations of the evolution of Leptin in heterothermic placentals show that this gene can be used as a molecular marker for understanding the evolution of heterothermy in bats.

When the common ancestor of bats originated (around $64 \mathrm{Mya}$ ), the worldwide climate was warm; however, long-term cooling occurred in some areas leading to temperate zones when the heterothermic bats (50 Mya-20 Mya) and most homeothermic bats (especially Old World fruit bats, 30 Mya-15 Mya) originated [3,22]. It is known that the length and depth of heterothermy are affected by body size, and that torpid periods increase with decreasing body size $[4,45]$. Most extant hibernating bats are small $(5-25 \mathrm{~g})$ and feed on insects $[1,4]$. As they require high energy expenditure to maintain a euthermic $\mathrm{T}_{\mathrm{b}}$, hibernation, as expressed in many temperate bats, is the most efficient way to save energy and survive on limited food [3]. In contrast, large tropical pteropodids $(>60 \mathrm{~g})$ appear to be homeothermic, whereas heterothermy seems restricted to a few small New- and OldWorld fruit bats $[38,46,47]$. The six species of Pteropodidae (28 Mya-18 Mya) used in our study are homeothermic and tropical/subtropical distribution. Abundant food, a mild climate and a large body size may explain the relaxed selection on Leptin of these homeothermic bats. Combining the evolutionary history of homeothermic bats and our molecular data, provides support for our hypothesis that heterothermic thermoregulation was lost in some tropical bats and adapted into hibernation in others. However, to further clearly assess how loss of heterothermy in bats, the heterothermic species of the Pteropodidae should be sampled.

The reconstruction of ancestral states and selection pressure analyses of Leptin strongly suggests that the ancestors of bats were heterothermic and that this ability became specialized ultimately resulting in hibernation especially in some temperate bats. Sliding window analysis showed that in heterothermic bats, most biofunctional domains of Leptin exon 3 have high $\omega$ ratios, especially in the AB loop and helix D (Figure 4). The total 29 amino acid variants specific to heterothermic bats with high MAPP scores were identified in six regions of Leptin exon 3 (Table S6). A few studies have analyzed the functional regions and determined the binding sites of Leptin with the receptors, and 10 of 22 binding sites of Leptin located in exon 3, including 7 in the functionally significant segment 85-119 [48,49,50,51]. Here, six of 29 sites are in the segment $85-119$ and two of them are binding sites indicated that natural selection may affact the intereaction of Leptin with the receptors. In addition, in pika study [21], 17 of 20 sites driven by the cold stress located in five functional regions of exon 3, and four of them were identical with our study (Table S6). More sites and more functional regions located suggessed the selected stress may be stronger in heterothermic bats. These results prove that natural selection may have had a large functional effect on Leptin of heterothermic bats, by stabilizing the conformation and binding with Leptin receptors, perhaps for reducing thermal sensitivity $[35,48,49,52,53]$.

As the sequence is the functional basis of protein, the substitutions of nucleotides or amino acids may lead to the functional change. We were able to validate that amino acid substitutions affect the function of heterothermic bat Leptin, by expressing two Leptin proteins of Miniopterus fuliginosus (hibernator) and Rousettus leschenaultii (homeothermy) in E. coli cells and measuring the lipolytic activity assay in vitro. The results of the MTT and LDH assays are consistent and also identical with that of our previous study on Leptin of Rhinolophus ferrumequinum and $R$. leschenaultii [54]. Our data show that Leptin in heterothermic bats (M. fuliginosus and $R$. ferrumequinum) is more lipolytic than in homeothermic bats, and there is no significant difference between two heterothermic bats. All these data support the results of physiological and molecular evolutionary analyses. Rewarming from low $\mathrm{T}_{\mathrm{b}}$ during torpor requires a rapid change in energy supply, and the accurate and efficient mobilization of fat is a key functional component [55]. We showed that Leptin of hibernating bats is more efficient and active than that of homeothermic bats with regard to lipolysis, and this will meet the physiological needs of a rapid rise in energy production. This is critically important because at the low $\mathrm{T}_{\mathrm{b}}$ during torpor the function of Leptin will be greatly reduced, but a rapid rise in lipolysis for energy production is required during rewarming at the end of a torpor bout. However, the coadaptation between interacting amino acid sites of Leptin and the receptors, and pleiotropy of Leptin evolution need to be further explored.

Our physiological study and molecular data, together with the evolutionary history of the Chiroptera, suggest that the ancestors of bats were heterothermic. Following continental drift and the migration of bats, and changes in climate and food habits, bats diverged and became distributed throughout most of the world. Heterothermy became specialized allowing hibernation in predominantly in temperate bats and was lost in some tropical bats, although many bat species still retained the ancestral ability to employ daily torpor. Importantly, the evolution of Leptin in bats was driven by natural selection (positive/relaxed selection) and played an important role in the evolution of bat thermoregulation.

\section{Supporting Information}

Text S1

(DOC)

Figure S1 Ancestral reconstruction of binary data (homeothermy vs. heterothermy. Branches show homeothermic (white) and heterothermic (black) families. ?: some species of this family are likely heterothermic. For details see Figure 2. (TIF)

Figure S2 Gene tree of Leptin. The phylogenetic gene tree of Leptin, based on nucleotide sequences of Leptin complete CDS, used for the codon maximum likelihood analysis. The $\omega$ ratios and numbers of non-synonymous substitution $(\mathrm{N} * \mathrm{dN})$ and synonymous substitution $(\mathrm{S} * \mathrm{dS})$ of some specific lineages were shown in bold and in bracket. The scale bar of " 0.1 " means 0.1 nucleotide substitution per site. [T], torpor; $[\mathrm{H}]$, hibernator. Lineages with $\omega>1$ were shown in red and the Pika lineage as control was shown in blue. ?: it is likely heterothermic.

(TIF)

Figure S3 SDS-PAGE and Western blot analyses of bat GST-Leptins. GST-Leptin proteins, $R$. leschenaultii (left) and M.fuliginosus (right), identified by 10\% SDS-PAGE (A)/(B) and Western blot with anti-human-Leptin antibody (C)/(D). GSTLeptin proteins were induced 0 and $4 \mathrm{~h}$ by IPTG $(0.3 \mathrm{mM})$ at $30^{\circ} \mathrm{C}$.

(TIF)

Table S1 The geographical distribution and use of torpor in bat species. (DOC)

Table S2 Primer combinations used for amplification of Leptin sequences from 19 bat species.

(DOG) 
Table S3 Accession number of the Leptin sequences used in this study.

(DOC)

Table S4 Likelihood values and parameter estimates for the Leptin complete CDS (32 species, 158 aa). $\omega: d_{\mathrm{N}} / d_{\mathrm{S}}$ ratio. $\ell$ : Log-likelihood ratio. Those in parentheses are presented for clarity only but are not free parameters.

(DOC)

Table S5 Likelihood values and parameter estimates for the Leptin exon 3 (46 species, 110 aa). $\omega: d_{\mathrm{N}} / d_{\mathrm{S}}$ ratio. $\ell$ : Log-likelihood ratio. Those in parentheses are presented for clarity only but are not free parameters.

(DOC)

\section{References}

1. Lyman CP (1970) Thermoregulation and metabolism in bats Wimsatt WA, ed. New York: Academic Press. pp 301-330.

2. Moss CF, Sinha SR (2003) Neurobiology of echolocation in bats. Curr Opin Neurobiol 13: 751-758.

3. Geiser F, Turbill C (2009) Hibernation and daily torpor minimize mammalian extinctions. Naturwissenschaften 96: 1235-1240.

4. Geiser F, Ruf T (1995) Hibernation versus daily torpor in mammals and birds: physiological variables and classification of torpor patterns. Physiological Zoology 68: 935-966.

5. Dausmann KH, Glos J, Ganzhorn JU, Heldmaier G (2004) Physiology: hibernation in a tropical primate. Nature 429: 825-826.

6. Srere HK, Wang LC, Martin SL (1992) Central role for differential gene expression in mammalian hibernation. Proc Natl Acad Sci U S A 89: 7119-7123.

7. Andrews MT, Squire TL, Bowen CM, Rollins MB (1998) Low-temperature carbon utilization is regulated by novel gene activity in the heart of a hibernating mammal. Proc Natl Acad Sci U S A 95: 8392-8397.

8. Storey KB, Storey JM (1990) Metabolic rate depression and biochemical adaptation in anaerobiosis, hibernation and estivation. Q Rev Biol 65: 145-174.

9. Cottrell J, Hogan C, Jain N, Nogal B, McWay M (2003) Modeling heat flows in a hibernating black bear. Available: http://hdl.handle.net/1813/137. pdf. Accessed 2011 Oct 25.

10. Jones KE, Purvis A, MacLarnon A, Bininda-Emonds OR, Simmons NB (2002) A phylogenetic supertree of the bats (Mammalia: Chiroptera). Biol Rev Camb Philos Soc 77: 223-259.

11. Geiser F, Stawski C (2011) Hibernation and torpor in tropical and subtropical bats in relation to energetics, extinctions, and the evolution of endothermy. Integr Comp Biol 51: 337-348.

12. Geiser F, Körtner G (2010) Hibernation and daily torpor in Australian mammals. Australian Zoologist 35: 204-215.

13. Ransome RD (1990) The natural history of hibernating bats (Christopher Helm Mammal Series). In Academic Press Inc.,U.S. pp 83-85.

14. Geiser F (1998) Evolution of daily torpor and hibernation in birds and mammals: importance of body size. Clin Exp Pharmacol Physiol 25: 736-739.

15. Zhang Y, Proenca R, Maffei M, Barone M, Leopold L, et al. (1994) Positional cloning of the mouse obese gene and its human homologue. Nature 372: 425-432.

16. Ormseth OA, Nicolson M, Pelleymounter MA, Boyer BB (1996) Leptin inhibits prehibernation hyperphagia and reduces body weight in arctic ground squirrels. Am J Physiol 271: R1775-1779.

17. Townsend KL, Kunz TH, Widmaier EP (2008) Changes in body mass, serum leptin, and mRNA levels of leptin receptor isoforms during the premigratory period in Myotis lucifugus. J Comp Physiol [B] 178: 217-223.

18. Kronfeld-Schor N, Richardson C, Silvia BA, Kunz TH, Widmaier EP (2000) Dissociation of leptin secretion and adiposity during prehibernatory fattening in little brown bats. Am J Physiol Regul Integr Comp Physiol 279: R1277-1281.

19. Florant GL, Porst H, Peiffer A, Hudachek SF, Pittman C, et al. (2004) Fat-cell mass, serum leptin and adiponectin changes during weight gain and loss in yellow-bellied marmots (Marmota flaviventris). J Comp Physiol [B] 174: 633-639.

20. Concannon P, Levac K, Rawson R, Tennant B, Bensadoun A (2001) Seasonal changes in serum leptin, food intake, and body weight in photoentrained woodchucks. Am J Physiol Regul Integr Comp Physiol 281: R951-959.

21. YangJ, Wang ZL, Zhao XQ, Wang de P, Qi de L, et al. (2008) Natural selection and adaptive evolution of leptin in the ochotona family driven by the cold environmental stress. PLoS ONE 3: e1472.

22. Teeling EC, Springer MS, Madsen O, Bates P, O'Brien S J, et al. (2005) A molecular phylogeny for bats illuminates biogeography and the fossil record. Science 307: 580-584.

23. Nishihara H, Hasegawa M, Okada N (2006) Pegasoferae, an unexpected mammalian clade revealed by tracking ancient retroposon insertions. Proc Natl Acad Sci U S A 103: 9929-9934.
Table S6 Summary of amino acid variants specifically in heterothermic bats identified by the MAPP analysis. (DOC)

\section{Acknowledgments}

We are grateful to Cullen Geiselman and Allyson L Walsh for samples, JP Zhang and JS Zhang for help with fieldwork, and HB Zhao and Y Liu for comments on an earlier version.

\section{Author Contributions}

Conceived and designed the experiments: LY SZ. Performed the experiments: LY X.Zhao BL X.Zuo LH GH. Analyzed the data: LY BL X.Zhao. Contributed reagents/materials/analysis tools: SZ BL. Wrote the paper: LY BL SZ SR GJ FG.

24. Maddison W, Maddison D (2007) Mesquite: A modular system for evolutionary analysis, version 2.5. Available: http://mesquiteproject.org/mesquite/download/ download.html. Accessed 2011 Oct 25

25. Pruitt KD, Tatusova T, Maglott DR (2005) NCBI Reference Sequence (RefSeq): a curated non-redundant sequence database of genomes, transcripts and proteins. Nucleic Acids Res 33: D501-504.

26. Withers PC, Thompson GG, Seymour RS (2000) Metabolic physiology of the north-western marsupial mole, Notoryctes caurinus (Marsupialia: Notoryctidae). Australian Journal of Zoology 48: 241-258.

27. Kumar S, Tamura K, Nei M (2004) MEGA3: Integrated software for Molecular Evolutionary Genetics Analysis and sequence alignment. Brief Bioinform 5: $150-163$.

28. Posada D, Crandall KA (1998) MODELTEST: testing the model of DNA substitution. Bioinformatics 14: 817-818.

29. Huelsenbeck JP, Ronquist F (2001) MRBAYES: Bayesian inference of phylogenetic trees. Bioinformatics 17: 754-755.

30. Yang Z (1997) PAML: a program package for phylogenetic analysis by maximum likelihood. Comput Appl Biosci 13: 555-556.

31. Nei M, Gojobori T (1986) Simple methods for estimating the numbers of synonymous and nonsynonymous nucleotide substitutions. Mol Biol Evol 3: 418-426.

32. Pride DT (2000) SWAAP - A tool for analyzing substitutions and similarity in multiple alignments. Available: http://www.bacteriamuseum.org/SWAAP/ SwaapPage.htm. Accessed 2011 Oct 25

33. Stone EA, Sidow A (2005) Physicochemical constraint violation by missense substitutions mediates impairment of protein function and disease severity. Genome Res 15: 978-986.

34. Ambati S, Kim HK, Yang JY, Lin J, Della-Fera MA, et al. (2007) Effects of leptin on apoptosis and adipogenesis in 3T3-L1 adipocytes. Biochem Pharmacol 73: $378-384$.

35. Grasso P, Leinung MC, Ingher SP, Lee DW (1997) In vivo effects of leptinrelated synthetic peptides on body weight and food intake in female ob/ob mice: localization of leptin activity to domains between amino acid residues 106-140. Endocrinology 138: 1413-1418.

36. Hemati N, Ross SE, Erickson RL, Groblewski GE, MacDougald OA (1997) Signaling pathways through which insulin regulates CCAAT/enhancer binding protein alpha (C/EBPalpha) phosphorylation and gene expression in 3T3-L1 adipocytes. Correlation with GLUT4 gene expression. J Biol Chem 272: 25913-25919.

37. Twente JW, Twente JA (1964) A hypothesis concerning the evolution of heterothermy in bats. Ann Acad Sci Fenn Series A Biol 1: 435-442.

38. Bartels W, Law BS, Geiser F (1998) Daily torpor and energetics in a tropical mammal, the northern blossom-bat Macroglossus minimus (Megachiroptera). J Comp Physiol [B] 168: 233-239.

39. Geiser F, Coburn D, Koertner G, Law B (1996) Thermoregulation, energy metabolism, and torpor in blossom-bats, Syconycteris australis (Megachiroptera). Journal of Zoology 239: 583-590.

40. McNab BK, Bonaccorso FJ (2001) The metabolism of New Guinean pteropodid bats. J Comp Physiol [B] 171: 201-214.

41. Geiser F (2008) Ontogeny and phylogeny of endothermy and torpor in mammals and birds. Comp Biochem Physiol A Mol Integr Physiol 150: $176-180$.

42. Berglund AC, Wallner B, Elofsson A, Liberles DA (2005) Tertiary windowing to detect positive diversifying selection. J Mol Evol 60: 499-504.

43. Benner SA, Caraco MD, Thomson JM, Gaucher EA (2002) Planetary biologypaleontological, geological, and molecular histories of life. Science 296: 864-868.

44. Gaucher EA, Mivamoto MM, Benner SA (2003) Evolutionary, structural and biochemical evidence for a new interaction site of the leptin obesity protein. Genetics 163: 1549-1553.

45. French AR (1985) Allometries of the duration of torpid and euthermic intervals during mammalian hibernation: a test of the theory of metabolic control of the 
timing of changes in body temperature. Journal of Comparative Physiology B 156: 13-19.

46. Audet D, Thomas DW (1997) Facultative hypothermia as a thermoregulatory strategy in the phyllostomid bats, Carollia perspicillata and Sturnira lilium. J Comp Physiol [B] 167: 146-152.

47. Kelm DH, von Helversen O (2007) How to budget metabolic energy: torpor in a small Neotropical mammal. J Comp Physiol [B] 177: 667-677.

48. Hiroike T, Higo J, Jingami H, Toh H (2000) Homology modeling of human leptin/leptin receptor complex. Biochem Biophys Res Commun 275: 154-158.

49. Grasso P, White DW, Tartaglia LA, Leinung MC, Lee DW (1999) Inhibitory effects of leptin-related synthetic peptide 116-130 on food intake and body weight gain in female $\mathrm{C} 57 \mathrm{BL} / 6 \mathrm{~J} \mathrm{ob} / \mathrm{ob}$ mice may not be mediated by peptide activation of the long isoform of the leptin receptor. Diabetes 48: 2204-2209.
50. Grasso P, Leinung MC, Lee DW (1999) Epitope mapping of secreted mouse leptin utilizing peripherally administered synthetic peptides. Regul Pept 85: 93-100.

51. Imagawa K, Numata Y, Katsuura G, Sakaguchi I, Morita A, et al. (1998) Structure-function studies of human leptin. J Biol Chem 273: 35245-35249.

52. Lee GH, Proenca R, Montez JM, Carroll KM, Darvishzadeh JG, et al. (1996) Abnormal splicing of the leptin receptor in diabetic mice. Nature 379: 632-635.

53. Zhang F, Basinski MB, Beals JM, Briggs SL, Churgay LM, et al. (1997) Crystal structure of the obese protein leptin-E100. Nature 387: 206-209.

54. He L, Pan Y, He G, Lin B, Liao CC, et al. (2010) Structural and functional studies of leptins from hibernating and non-hibernating bats. Gen Comp Endocrinol 168: 29-35.

55. Carey HV, Andrews MT, Martin SL (2003) Mammalian hibernation: cellular and molecular responses to depressed metabolism and low temperature. Physiol Rev 83: 1153-1181. 